\title{
Global Stability of Approximation for Exponential Attractors
}

\author{
By
}

\author{
Masashi AIDA and Atsushi YAGI ${ }^{1}$ \\ (Osaka University, Japan)
}

\begin{abstract}
This paper is concerned with the initial value problem for some diffusion system which describes the process of a pattern formation of biological individuals by chemotaixis and growth. In the paper Osaki et al. [13], exponential attractors have been constructed for the dynamical system determined by this problem. The exponential attractor is one of limit sets which is a positively invariant compact set with finite fractal dimension and which attracts every trajectory in an exponential rate. In this paper we study another feature of exponential attractors, that is we show that the approximate solution also gets close to the exponential attractor in an exponential rate and remains in its neighborhood forever. Our methods are available to any other exponential attractors determined by interaction-diffusion systems.

Key Words and Phrases. Exponential attractors, Global stability under approximation, Chemotaxis-growth system.

2000 Mathematics Subject Classification Numbers. Primary 37L15; Secondary 65P99, 92D25.
\end{abstract}

\section{Introduction}

We are concerned with the initial value problem of the following chemotaxis-growth system

$$
\left\{\begin{array}{l}
\frac{\partial u}{\partial t}=a \Delta u-\nabla \cdot\{u \nabla \chi(\rho)\}+f(u) \quad \text { in } \Omega \times(0, \infty) \\
\frac{\partial \rho}{\partial t}=b \Delta \rho-c \rho+d u \quad \text { in } \Omega \times(0, \infty) \\
\frac{\partial u}{\partial n}=\frac{\partial \rho}{\partial n}=0 \quad \text { on } \partial \Omega \times(0, \infty) \\
u(x, 0)=u_{0}(x), \rho(x, 0)=\rho_{0}(x) \text { in } \Omega
\end{array}\right.
$$

This problem arises in mathematical biology, here $u(x, t)$ and $\rho(x, t)$ denote the population density of biological individuals and the concentration of chemical substance at a position $x \in \Omega \subset \boldsymbol{R}^{2}$ and time $t \in[0, \infty)$, respectively. The mobility of individuals consists of random walking and chemotaxis. The constants

\footnotetext{
1 Partially supported by Grant-in-Aid for Scientific Research (No. 14540204) by Japan Society for
} the Promotion of Science. 
$a>0$ and $b>0$ are the diffusion rates of $u$ and $\rho$, respectively; $c>0$ and $d>0$ are the degradation and production rates of $\rho$, respectively. The function $\chi(\rho)$ is a sensitivity function due to chemotaxis. The function $f(u)$ denotes a growth rate of $u$.

This model was presented by Mimura and Tsujikawa [8] in order to study theoretically the complex spatio-temporal colony patterns of $E$. coli which were observed experimentally by Budrene and Berg [4]. Among other models [3, 6, $7,10,17]$ etc., theirs is rather simple in the sense that it incorporates only four effects: diffusion, chemotaxis, production of chemical substance, and growth.

In the paper Osaki et al. [13] we have already studied the global existence of solutions and also have succeeded in constructing an exponential attractor for the dynamical system determined from (1.1). The notion of exponential attractors was introduced in Eden, Foias, Nicolaenko, and Temam [22]. It is one of limit sets of dynamical systems in infinite-dimensional phase spaces, see a summary in Section 2. If the attractor exists, it contains the global attractor as a subset, has finite fractal dimension, and attracts all trajectories in an exponential rate. It is known that, if the semigroup corresponding to a dynamical system satisfies some favorable property (the squeezing property [22], the compact smoothing property [5], or some others), then one can construct the exponential attractors.

Formation of the colony patterns by chemotaxis is known as a prototype of various phenomena of Self-Organization, cf. [23, 24]. According to description by Synergetics due to Haken [23], the chemical substance plays a role of the conductor which leads the individuals and is itself produced by them cooperatively. Fractal dimension of the exponential attractor then corresponds to the number of active modes, and its attraction of the trajectories corresponds to a reduction of the degrees of freedom in the process of pattern formation which is called the slaving principle.

For observing profiles of the solutions which evolve in the exponential attractor and knowing a structure of the attractor, the numerical method plays a chief role, cf. $[8,9,15,16]$. In this paper we are concerned with a feature which the exponential attractor possesses in global stability of approximation. In fact we shall show that, under time local convergence of spatially descretized approximate semigroups to the continuous semigroup, all approximate solutions get close to the exponential attractor in an exponential rate, and then they are trapped in its neighborhood forever. In general, approximate solutions draw apart from their true solutions; but when an exponential attractor exists, they remain in its neighborhood and continue to approximate some trajectories which lie in the exponential attractor. In this sense, our results might guarantee global reliability of numerical computation.

A summary of exponential attractors is made in Section 2. In Section 3 
we derive from time local convergence of spatially descretized semigroups their convergence to the exponential attractor. Since the result is described in a general form, it is applicable not only to (1.1) also to many other nonlinear diffusion systems (cf. [21]). Section 4 is devoted to investigating the local convergence of approximate semigroups. An abstract parabolic evolution equation and its semidiscretized equation are considered. Under suitable assumptions we establish time local convergence. All these abstract results are applied in Section 5 to the chemotaxis-growth system (1.1), and the main result of the paper is obtained.

Notations. Let $X$ be a Banach space with norm $\|\cdot\|_{X}$ and $\mathscr{X}$ be a subset of $X . \quad \mathscr{X}$ is a metric space with the induced distance $d(U, V)=\|U-V\|_{X}$ $(U, V \in \mathscr{X})$. For $U \in \mathscr{X}$ and a set $B \subset \mathscr{X}, d(U, B)$ is defined by $d(U, B)=$ $\inf _{V \in B} d(U, V)$. For two sets $B_{1}, B_{2} \subset \mathscr{X}$, their distance $d\left(B_{1}, B_{2}\right)$ is defined by $d\left(B_{1}, B_{2}\right)=\max \left\{h\left(B_{1}, B_{2}\right), h\left(B_{2}, B_{1}\right)\right\}$, where $h\left(B_{1}, B_{2}\right)$ denotes the Hausdorff pseudodistance given by

$$
h\left(B_{1}, B_{2}\right)=\sup _{U \in B_{1}} d\left(U, B_{2}\right)=\sup _{U \in B_{1}} \inf _{V \in B_{2}} d(U, V) .
$$

For two Banach spaces $X$ and $Y, \mathscr{L}(X, Y)$ denotes the space of bounded linear operators from $X$ into $Y$ with the uniform operator norm $\|\cdot\|_{\mathscr{L}(X, Y)}$. $\mathscr{L}(X, X)$ is abbreviated as $\mathscr{L}(X)$.

Let $X$ be a Banach space and let $I$ be an interval. $\mathscr{C}(I ; X), \mathscr{C}^{\theta}(I ; X)$ $(0<\theta<1)$ and $\mathscr{C}^{1}(I ; X)$ denote the space of $X$-valued continuous functions, Hölder continuous functions with exponent $\theta$, and continuously differentiable functions equipped with the usual function norms, respectively. $\mathscr{B}(I ; X)$ is the space of $X$-valued bounded functions (not necessarily measurable) equipped with the norm $\|f\|_{\mathscr{B}}=\sup _{t \in I}\|f(t)\|_{X}$. Finally, $\mathscr{F} \gamma, \sigma((0, T] ; X) \quad(0<\sigma<\gamma \leq 1)$ is a space of $X$-valued Hölder continuous functions on $(0, T]$ which satisfy

$$
\sup _{0<t \leq T} t^{1-\gamma}\|F(t)\|_{X}<\infty \quad \text { and } \quad \sup _{0<s<t \leq T} \frac{s^{1-\gamma+\sigma}\|F(t)-F(s)\|_{X}}{(t-s)^{\sigma}}<\infty .
$$

Its norm is defined as usual by

$$
\|F\|_{\mathscr{F} \gamma, \sigma}=\sup _{0<t \leq T} t^{1-\gamma}\|F(t)\|_{X}+\sup _{0<s<t \leq T} \frac{s^{1-\gamma+\sigma}\|F(t)-F(s)\|_{X}}{(t-s)^{\sigma}} .
$$

\section{Summary of exponential attractors}

Let $X$ be a Banach space with norm $\|\cdot\|_{X}$ and let $\mathscr{X}$ be a subset of $X$. $\mathscr{X}$ is a metric space with the induced distance $d(U, V)=\|U-V\|_{X}(U, V \in \mathscr{X})$. A family $\{S(t)\}_{0 \leq t<\infty}$ of nonlinear operators on $\mathscr{X}$ is called a semigroup if 


$$
S(t) S(s)=S(t+s) \quad \text { for } 0 \leq s, t<\infty, \quad \text { and } \quad S(0)=I .
$$

When a semigroup enjoys the following property, $S(t)$ is called a continuous semigroup:

(2.1) $G\left(t, U_{0}\right)=S(t) U_{0}$ is a continuous mapping from $[0, \infty) \times \mathscr{X}$ into $\mathscr{X}$.

Let $\left\{U_{n}\right\}_{n=0,1,2, \ldots}$ be a sequence of $\mathscr{X}$ which is convergent to an element $\bar{U} \in \mathscr{X}$. Then, for any $0<T<\infty$, the set $[0, T] \times\left\{U_{0}, U_{1}, U_{2}, \ldots, \bar{U}\right\}$ is a compact set of $[0, \infty) \times \mathscr{X}$; therefore, under $(2.1), G$ is uniformly continuous on the compact set. Hence, if $S(t)$ is a continuous semigroup and if $U_{n} \rightarrow \bar{U}$ in $\mathscr{X}$, then

$$
\sup _{0 \leq t \leq T} d\left(S(t) U_{n}, S(t) \bar{U}\right) \rightarrow 0, \quad \text { as } n \rightarrow \infty
$$

for any finite $0<T<\infty$.

Let $S(t)$ be a continuous semigroup on $\mathscr{X}$. For each $U_{0} \in \mathscr{X}$, the continuous function $S(\cdot) U_{0}$ is called the trajectory starting from $U_{0}$. The family of all such trajectories are denoted by $(S(t), \mathscr{X}, X)$, and is called a dynamical system determined by the semigroup $S(t)$ on the phase space $\mathscr{X}$ in the universal space $X$.

Let $(S(t), \mathscr{X}, X)$ be a dynamical system. A set $\mathscr{A} \subset \mathscr{X}$ is called a positively invariant set of $S(t)$ if

$$
S(t) \mathscr{A} \subset \mathscr{A} \quad \text { for every } t \geq 0,
$$

and is called an invariant set of $S(t)$ if

$$
S(t) \mathscr{A}=\mathscr{A} \quad \text { for every } t \geq 0 .
$$

A set $\mathscr{A} \subset \mathscr{X}$ is said to attract a set $B \subset \mathscr{X}$ if

$$
\lim _{t \rightarrow \infty} h(S(t) B, \mathscr{A})=0
$$

where $h(\cdot, \cdot)$ denotes the pseudodistance given by (1.2). As is easily seen, this means that for any $\varepsilon>0$, there exists some $T>0$ such that

$$
S(t) B \subset W_{\varepsilon}(\mathscr{A}) \quad \text { for every } t \geq T,
$$

where $W_{\varepsilon}(\mathscr{A})$ denotes the $\varepsilon$-neighborhood of $\mathscr{A}$.

An invariant set $\mathscr{A}$ of $S(t)$ is called an attractor of the dynamical system if $\mathscr{A}$ attracts some open neighborhood of $\mathscr{A}$ in $\mathscr{X}$, namely $\lim _{t \rightarrow \infty} h(S(t) W, \mathscr{A})=$ 0 for some open set $W \supset \mathscr{A}$.

An attractor $\mathscr{A}$ is called a global attractor if

(1) $\mathscr{A}$ is a compact set of $X$;

(2) $\mathscr{A}$ attracts every bounded set of $\mathscr{X}$, namely

$$
\lim _{t \rightarrow \infty} h(S(t) B, \mathscr{A})=0 \quad \text { for every bounded set } B \subset \mathscr{X} .
$$


In this paper we are interested in limit sets which attract every bounded set exponentially. Consider a dynamical system $(S(t), \mathscr{X}, X)$ determined from a continuous semigroup $S(t)$ on $\mathscr{X}$. We assume that

$$
\mathscr{X} \text { is a compact set of } X \text {. }
$$

This assumption is not so restrictive in our application. Indeed, we can see for the dynamical system determined from (1.1) that there exists a compact and positively invariant set to which every trajectory enters after a certain time (depending on the initial point $U_{0}$ ). This means in the view point of limit sets that the dynamical system is reduced to a smaller system in which the phase space is a compact set.

Under (2.3) it is easily verified that the set $\mathscr{A}$ given by

$$
\mathscr{A}=\bigcap_{t \geq 0} S(t) \mathscr{X}
$$

is a global attractor of $(S(t), \mathscr{X}, X)$. In fact, (2.3) yields that $\mathscr{A}$ is a nonempty compact set of $\mathscr{X}$. Furthermore, it is verified that $U \in \mathscr{A}$ if and only if there exist a time sequence $t_{n} \rightarrow \infty$ and a sequence of elements $U_{n} \in \mathscr{X}$ such that $S\left(t_{n}\right) U_{n}$ converges to $U$; from this characterization of the element of $\mathscr{A}, \mathscr{A}$ is shown to be an invariant set of $S(t)$. We next verify that $\mathscr{A}$ attracts the whole phase space $\mathscr{X}$. Suppose the contrary; then, there would exist some $\alpha>0$ such that, for any $T>0, h(S(t) \mathscr{X}, \mathscr{A})>\alpha$ with some $t \geq T$. This then implies that there exist a time sequence $t_{n} \rightarrow \infty$ and a sequence $U_{n} \in \mathscr{X}$ such that $d\left(S\left(t_{n}\right) U_{n}, \mathscr{A}\right) \geq \alpha$. By (2.3), $\left\{S\left(t_{n}\right) U_{n}\right\}_{n \geq 0}$ contains some subsequence which is convergent to $\bar{U} \in \mathscr{X}$. Clearly, $d(\bar{U}, \mathscr{A}) \geq \alpha$; but, since $\bar{U} \in \mathscr{A}$, this is a contradiction. Hence, $\mathscr{A}$ attracts the whole space $\mathscr{X}$.

Under (2.3), a subset $\mathscr{M}$ such that $\mathscr{A} \subset \mathscr{M} \subset \mathscr{X}$ is called an exponential attractor of $(S(t), \mathscr{X}, X)$ if

(1) $\mathscr{M}$ is a compact set of $X$ with finite fractal dimension $d_{F}(\mathscr{M})$ in $X$;

(2) $\mathscr{M}$ is a positively invariant set of $S(t), S(t) \mathscr{M} \subset \mathscr{M}$ for every $0 \leq$ $t<\infty$;

(3) $\mathscr{M}$ attracts $\mathscr{X}$ exponentially in the sense that

$$
h(S(t) \mathscr{X}, \mathscr{M}) \leq M e^{-\beta t}, \quad 0 \leq t<\infty
$$

with some constant $M>0$ and some exponent $\beta>0$.

By some abuse of terminology we call $\mathscr{M}$ an exponential attractor, but $\mathscr{M}$ may not be an attractor in an accurate sense. The attractor must be an invariant set (see (2.2)).

A few general results are already known concerning construction of exponential attractors. When $X$ is a Hilbert space, Eden, Foias, Nicolaenko and Temam [22] have shown that under the Lipschitz condition of the mapping 
$G\left(t, U_{0}\right)=S(t) U_{0}$ and the squeezing property described below, one can construct an exponential attractor of $(S(t), \mathscr{X}, X)$. When $X$ is a Hilbert space, the following property of $S(t)$ is called the squeezing property. For some $0<\delta<\frac{1}{4}$ and some fixed time $t^{*}>0$, there exists an orthogonal projection $P$ of finite rank such that either

$$
\left\|S\left(t^{*}\right) U-S\left(t^{*}\right) V\right\|_{X} \leq \delta\|U-V\|_{X}
$$

or

$$
\left\|(1-P)\left\{S\left(t^{*}\right) U-S\left(t^{*}\right) V\right\}\right\|_{X} \leq\left\|P\left\{S\left(t^{*}\right) U-S\left(t^{*}\right) V\right\}\right\|_{X}
$$

holds for each pair of $U, V \in \mathscr{X}$.

More recently Efendiev, Miranville and Zelik [5] presented a different method which is available even when $X$ is a Banach space. Indeed, they have shown that the squeezing property is replaced by the compact smoothing property. The following property is called the compact smoothing property of $S(t)$. For some fixed time $t^{*}>0$,

$$
\left\|S\left(t^{*}\right) U-S\left(t^{*}\right) V\right\|_{Y} \leq L\|U-V\|_{X}, \quad U, V \in \mathscr{X}
$$

holds with another Banach space $Y$ which is compactly embedded in $X$.

In applications it seems to be easier to verify (2.7) than (2.5) and (2.6) (cf. [2]). We must remark, however, that two exponential attractors constructed by these methods may be quite different and therefore the fractal dimensions may be greatly different, because we have no uniqueness of exponential attractors in general.

\section{Global stability of approximation}

Consider a dynamical system $(S(t), \mathscr{X}, X)$ determined from a continuous semigroup $S(t)$ on a compact phase space $\mathscr{X} \subset X$. We assume that the dynamical system possesses an exponential attractor $\mathscr{M}$ with rate (2.4).

Let $\left\{\mathscr{X}_{\xi}\right\}_{0<\xi \leq \xi_{0}}$ be a family of approximate phase spaces of $\mathscr{X}$ such that

$$
\mathscr{X}_{\xi} \subset \mathscr{X}, \quad 0<\xi \leq \xi_{0} .
$$

Let

$$
\left\{S_{\xi}(t)\right\}_{0 \leq t<\infty} \text { be a semigroup acting on } \mathscr{X}_{\xi}, \quad 0<\xi \leq \xi_{0},
$$

(not necessarily continuous) which is assumed to approximate $S(t)$ as $\xi \rightarrow 0$ in the following sense. For each fixed $T>0$, it holds that

$$
\lim _{\xi \rightarrow 0} Q_{\xi}(T)=0, \quad \text { where } Q_{\xi}(T)=\sup _{\hat{U}_{\xi} \in \mathscr{X}_{\xi}} \sup _{0 \leq t \leq T} d\left(S_{\xi}(t) \hat{U}_{\xi}, S(t) \hat{U}_{\xi}\right) .
$$

We then verify the following theorems. 
Theorem 3.1. Under (2.4), (3.1), (3.2) and (3.3), let $\hat{U}_{\xi} \in \mathscr{X}_{\xi}$ and let $0<$ $T<\infty$ be arbitrary time. Then,

$$
d\left(S_{\xi}(t) \hat{U}_{\xi}, \mathscr{M}\right) \leq \begin{cases}M e^{-\beta t}+Q_{\xi}(T) & \text { for } 0 \leq t \leq T, \\ M e^{-\beta T}+Q_{\xi}(T) & \text { for } T \leq t<\infty .\end{cases}
$$

Proof. Let $\hat{U}_{\xi} \in \mathscr{X}_{\xi}$ and let $0 \leq t \leq T$. For any $U \in \mathscr{M}$,

$$
d\left(S_{\xi}(t) \hat{U}_{\xi}, \mathscr{M}\right) \leq d\left(S_{\xi}(t) \hat{U}_{\xi}, U\right) \leq d\left(S_{\xi}(t) \hat{U}_{\xi}, S(t) \hat{U}_{\xi}\right)+d\left(S(t) \hat{U}_{\xi}, U\right) .
$$

Here we see from (3.3) that

$$
d\left(S_{\xi}(t) \hat{U}_{\xi}, S(t) \hat{U}_{\xi}\right) \leq Q_{\xi}(T)
$$

In addition, for any $\varepsilon>0$, we can take some element $U \in \mathscr{M}$ such that

$$
d\left(S(t) \hat{U}_{\xi}, U\right) \leq d\left(S(t) \hat{U}_{\xi}, \mathscr{M}\right)+\varepsilon .
$$

By (1.2), (2.4), and (3.1),

$$
d\left(S(t) \hat{U}_{\xi}, \mathscr{M}\right) \leq h(S(t) \mathscr{X}, \mathscr{M}) \leq M e^{-\beta t} .
$$

Hence the estimate (3.4) is verified for the case when $0 \leq t \leq T$.

Let $\hat{U}_{\xi} \in \mathscr{X}_{\xi}$ and let now $T \leq t<\infty$. Similarly, for any $U \in \mathscr{M}$,

$$
\begin{aligned}
d\left(S_{\xi}(t) \hat{U}_{\xi}, \mathscr{M}\right) \leq & d\left(S_{\xi}(t) \hat{U}_{\xi}, U\right) \\
\leq & d\left(S_{\xi}(T) S_{\xi}(t-T) \hat{U}_{\xi}, S(T) S_{\xi}(t-T) \hat{U}_{\xi}\right) \\
& +d\left(S(T) S_{\xi}(t-T) \hat{U}_{\xi}, U\right) .
\end{aligned}
$$

Here, by (3.2) and (3.3),

$$
d\left(S_{\xi}(T) S_{\xi}(t-T) \hat{U}_{\xi}, S(T) S_{\xi}(t-T) \hat{U}_{\xi}\right) \leq Q_{\xi}(T) .
$$

In addition, for any $\varepsilon>0$, we can take some element $U \in \mathscr{M}$ such that

$$
d\left(S(T) S_{\xi}(t-T) \hat{U}_{\xi}, U\right) \leq d\left(S(T) S_{\xi}(t-T) \hat{U}_{\xi}, \mathscr{M}\right)+\varepsilon .
$$

By (1.2), (2.4), (3.1) and (3.2),

$$
d\left(S(T) S_{\xi}(t-T) \hat{U}_{\xi}, \mathscr{M}\right) \leq h(S(T) \mathscr{X}, \mathscr{M}) \leq M e^{-\beta T} .
$$

Hence, the estimate (3.4) is valid for the case when $T \leq t<\infty$ also.

This theorem in turn yields some convergence of an attractor of $\left\{S_{\xi}(t)\right\}_{0 \leq t<\infty}$ to $\mathscr{M}$.

Theorem 3.2. Under (2.4), (3.1), (3.2) and (3.3), let

$$
\mathscr{A}_{\xi}=\bigcap_{t \geq 0} \overline{\bigcup_{s \geq t} S_{\xi}(s) \mathscr{X}_{\xi}} \text {. }
$$


Then, for arbitrary time $0<T<\infty$,

$$
h\left(\mathscr{A}_{\xi}, \mathscr{M}\right) \leq M e^{-\beta T}+Q_{\xi}(T), \quad 0<\xi \leq \xi_{0} .
$$

In particular, $\lim _{\xi \rightarrow 0} h(\mathscr{A} \xi, \mathscr{M})=0$.

Proof. Let $\hat{U}_{\xi} \in \mathscr{A}_{\xi}$. Then there exists a time sequence $t_{n} \rightarrow \infty$ and a sequence of elements $\hat{U}_{\xi, n} \in \mathscr{X}_{\xi}$ such that $S_{\xi}\left(t_{n}\right) \hat{U}_{\xi, n} \rightarrow \hat{U}_{\xi}$ as $n \rightarrow \infty$. In addition, there exists a sequence of elements $U_{n} \in \mathscr{M}$ such that

$$
d\left(S_{\xi}\left(t_{n}\right) \hat{U}_{\xi, n}, U_{n}\right) \leq d\left(S_{\xi}\left(t_{n}\right) \hat{U}_{\xi, n}, \mathscr{M}\right)+\frac{1}{n} .
$$

Using (3.4) we have

$$
d\left(S_{\xi}\left(t_{n}\right) \hat{U}_{\xi, n}, U_{n}\right) \leq M e^{-\beta T}+Q_{\xi}(T)+\frac{1}{n}
$$

for sufficiently large $n$ such that $t_{n} \geq T$. Since we can assume that $U_{n}$ is also convergent to some element $\bar{U} \in \mathscr{M}$, it is obtained that

$$
d\left(\hat{U}_{\xi}, \mathscr{M}\right) \leq d\left(\hat{U}_{\xi}, \bar{U}\right) \leq M e^{-\beta T}+Q_{\xi}(T) .
$$

Since $\hat{U}_{\xi}$ can be taken arbitrarily in $\mathscr{A}_{\xi}$, we have proved the first assertion.

From (3.5) we have $\limsup _{\xi \rightarrow 0} h\left(\mathscr{A}_{\xi}, \mathscr{M}\right) \leq M e^{-\beta T}$ with arbitrary time $0<T<\infty$. Hence the second assertion is also verified.

From Theorem 3.1 we verify the following fact. Every trajectory $S_{\xi}(t) \hat{U}_{\xi}$, $\hat{U}_{\xi} \in \mathscr{X}_{\xi}$, approaches to $\mathscr{M}$ asymptotically as $t \rightarrow \infty$, and stays in a neighborhood of $\mathscr{M}$ forever, provided $\xi>0$ is sufficiently small. Indeed, let $\varepsilon \in(0,2 M)$ be any number. Fix $T>0$ so that $M e^{-\beta T}=\frac{\varepsilon}{2}$, namely $T=$ $\frac{1}{\beta} \log \left(\frac{2 M}{\varepsilon}\right)$, and let $\xi_{1}>0$ be sufficiently small such that, in view of (3.3), $Q_{\xi}(T) \leq \frac{\varepsilon}{2}$ for every $\xi \in\left(0, \xi_{1}\right]$. Then, for any $0<\xi \leq \xi_{1}$ and any $\hat{U}_{\xi} \in \mathscr{X}_{\xi}$, we observe that

$$
d\left(S_{\xi}(t) \hat{U}_{\xi}, \mathscr{M}\right) \leq \varepsilon \quad \text { for every } t \geq T
$$

\section{Local convergence of approximation}

In this section we shall investigate sufficient conditions which imply the condition (3.3). For this purpose we confine ourselves to the case where $S(t)$ is defined by the Cauchy problem for a quasilinear abstract evolution equation, and treat a semi discretization scheme for the spatial variable.

We consider the Cauchy problem for a quasilinear abstract evolution equation 


$$
\left\{\begin{array}{l}
\frac{d U}{d t}+A(U) U=F(U), \quad 0<t<\infty, \\
U(0)=U_{0}
\end{array}\right.
$$

in a Banach space $X$. Let $Y$ be a second Banach space continuously embedded in $X$ and let $K$ be an open ball of $Y$ such that

$$
K=\left\{U \in Y ;\|U\|_{Y}<R\right\}, \quad 0<R<\infty .
$$

For each $U \in K, A(U)$ is a densely defined closed linear operator in $X$ with domain $\mathscr{D}(A(U))$ which is independent of $U \in K, F$ is a nonlinear operator from $K$ into $X$, and $U_{0}$ is an initial value at least in $K$.

We also consider the Cauchy problem for a semi discretization equation

$$
\left\{\begin{array}{l}
\frac{d \hat{U}_{\xi}}{d t}+A_{\xi}\left(\hat{U}_{\xi}\right) \hat{U}_{\xi}=F_{\xi}\left(\hat{U}_{\xi}\right), \quad 0<t<\infty, \\
\hat{U}_{\xi}(0)=\hat{U}_{0, \xi}
\end{array}\right.
$$

in $X_{\xi}$, where $\left\{X_{\xi}\right\}_{0<\xi \leq \xi_{0}}$ is a family of approximate spaces of $X$ such that $X_{\xi} \subset Y$. For $U \in K, A_{\xi}(U)$ is an approximate operator of $A(U)$ which is a bounded linear operator on $X_{\xi}, F_{\xi}$ is an approximate operator of $F$ from $K \cap X_{\xi}$ into $X_{\xi}$, and $\hat{U}_{\xi, 0}$ is an initial value in $K \cap X_{\xi}$.

Structural Assumptions. The following conditions are assumed as structural assumptions. For the spectral set $\sigma(A(U))$,

$$
\sigma(A(U)) \subset \Sigma=\{\lambda \in \mathbf{C} ;|\arg \lambda|<\phi\}, \quad 0<\phi<\frac{\pi}{2},
$$

and the resolvent satisfies

$$
\left\|(\lambda-A(U))^{-1}\right\|_{\mathscr{L}(X)} \leq \frac{M}{|\lambda|+1}, \quad \lambda \notin \Sigma, U \in K .
$$

The domain $\mathscr{D}(A(U))$ is independent of $U$, and the Lipschitz condition

$$
\left\|A(U)\left\{A(U)^{-1}-A(V)^{-1}\right\}\right\|_{\mathscr{L}(X)} \leq N\|U-V\|_{Y}, \quad U, V \in K
$$

is satisfied.

The function $F$ also satisfies a Lipschitz condition

$$
\|F(U)-F(V)\|_{X} \leq L\|U-V\|_{Y}, \quad U, V \in K .
$$

With some exponent $0<\alpha<1, \mathscr{D}\left(A(U)^{\alpha}\right) \subset Y$ holds for every $U \in K$ with the estimate

$$
\|\tilde{U}\|_{Y} \leq D\left\|A(U)^{\alpha} \tilde{U}\right\|_{X}, \quad \tilde{U} \in \mathscr{D}\left(A(U)^{\alpha}\right), U \in K .
$$


We consider a family $\left\{X_{\xi}\right\}_{0<\xi \leq \xi_{0}}$ of finite dimensional subspaces of $X$ such that $X_{\xi} \subset Y, X_{\xi}$ being equipped with the norm of $X$. But when $X_{\xi}$ are equipped with the induced norm of $Y$, those spaces will be denoted by $Y_{\xi}$. For each $0<\xi \leq \xi_{0}, P_{\xi}$ denotes a projection from $X$ onto $X_{\xi}$. We assume that $P_{\xi}$ satisfies a uniform estimate $\left\|P_{\xi}\right\|_{\mathscr{L}\left(X, X_{\xi}\right)} \leq C, C>0$ being independent of $\xi$.

For each $U \in K$, an approximate operator $A_{\xi}(U)$ of $A(U)$ is defined as a linear operator from $X_{\xi}$ into itself. The operators $A_{\xi}(U)$ are assumed to satisfy the following various conditions uniformly, that is all the constants below are independent of the parameter $\xi$. As for the operator norms of $A_{\xi}(U)$,

$$
\left\|A_{\xi}(U)\right\|_{\mathscr{L}\left(X_{\xi}\right)} \leq \hat{C} O_{\xi}^{-1}, \quad U \in K .
$$

Here and in what follows, $O_{\xi}$ denotes the order of scheme which is a function of $\xi$ tending to 0 as $\xi \rightarrow 0$, and is specifically determined from the scheme used. For the spectral set $\sigma\left(A_{\xi}(U)\right)$,

$$
\sigma\left(A_{\xi}(U)\right) \subset \Sigma=\{\lambda \in \mathbf{C} ;|\arg \lambda|<\phi\}, \quad 0<\phi<\frac{\pi}{2},
$$

and the resolvent satisfies

$$
\left\|\left(\lambda-A_{\xi}(U)\right)^{-1}\right\|_{\mathscr{L}\left(X_{\xi}\right)} \leq \frac{\hat{M}}{|\lambda|+1}, \quad \lambda \notin \Sigma, U \in K .
$$

The following Lipschitz condition

$$
\left\|A_{\xi}(U)\left\{A_{\xi}(U)^{-1}-A_{\xi}(V)^{-1}\right\}\right\|_{\mathscr{L}\left(X_{\xi}\right)} \leq \hat{N}\|U-V\|_{Y}, \quad U, V \in K
$$

is satisfied.

With the same exponent $\alpha$ as in (4.6),

$$
\left\|\hat{U}_{\xi}\right\|_{Y_{\xi}} \leq \hat{D}\left\|A_{\xi}(U)^{\alpha} \hat{U}_{\xi}\right\|_{X_{\xi}}, \quad \hat{U}_{\xi} \in Y_{\xi}, U \in K
$$

As $\xi \rightarrow 0, P_{\xi}$ converges to the identity operator in the following sense

$$
\left\|\left\{1-P_{\xi}\right\} A(U)^{-1}\right\|_{\mathscr{L}(X)} \leq C O_{\xi}, \quad U \in K .
$$

Furthermore, let $R_{\xi}(U)=A_{\xi}(U)^{-1} P_{\xi} A(U), U \in K$, be the Ritz projection. The Ritz projection is assumed to converge to the identity operator in the following sense

$$
\begin{gathered}
\left\|\left\{1-R_{\xi}(U)\right\} A(U)^{-1}\right\|_{\mathscr{L}(X)} \leq C O_{\xi}, \quad U \in K, \\
\left\|\left\{R_{\xi}(U)-R_{\xi}(V)\right\} A(V)^{-1}\right\|_{\mathscr{L}(X)} \leq C O_{\xi}\|U-V\|_{Y}, \quad U, V \in K .
\end{gathered}
$$

Finally, $F_{\xi}(U)$ is defined as $F_{\xi}(U)=P_{\xi} F(U), U \in K$. 
Solutions to (4.1). The initial value $U_{0}$ is assumed to satisfy the basic estimate $\left\|U_{0}\right\|_{Y}<R$ and the compatibility condition

$$
U_{0} \in \mathscr{D}\left(A\left(U_{0}\right)^{\gamma}\right)
$$

with some exponent $\gamma$ such that

$$
0<\alpha<\gamma \leq 1 \quad \text { and } \quad \frac{1}{2}<\gamma .
$$

Then, it is already known by $[20,21]$ that (4.1) possesses a unique local solution in the function space

$$
\left\{\begin{array}{l}
U \in \mathscr{C}^{\gamma-\alpha}\left(\left[0, T_{U_{0}}\right] ; Y\right) \cap \mathscr{C}^{1}\left(\left(0, T_{U_{0}}\right] ; X\right) \\
A(U)^{\gamma} U \in \mathscr{B}\left(\left[0, T_{U_{0}}\right] ; X\right), \quad A(U) U \in \mathscr{F}^{\gamma, \sigma}\left(\left(0, T_{U_{0}}\right] ; X\right) .
\end{array}\right.
$$

Furthermore, if $U$ satisfies a suitable a priori estimates we can extend the local solution over the interval $\left[0, T_{U_{0}}\right]$. On the basis of these facts, we assume that on some fixed interval $[0, T],(4.1)$ possesses a solution in the same function spaces (4.15) with $\left[0, T_{U_{0}}\right]$ replaced by $[0, T]$.

Stability Conditions. Let $\left\{\hat{U}_{0, \xi}\right\}_{0<\xi \leq \xi_{0}}$ be a family of initial values such that

$$
\hat{U}_{0, \xi} \in X_{\xi}, \quad 0<\xi \leq \xi_{0}
$$

which approximate $U_{0}$ with the estimate $\left\|\hat{U}_{0, \xi}\right\|_{Y}<R$. We assume the existence of solution to (4.2) on the same interval $[0, T]$ as for (4.1). That is, for every $0<\xi \leq \xi_{0}$, there exists a solution $\hat{U}_{\xi}$ such that $\hat{U}_{\xi} \in \mathscr{C}^{1}\left([0, T] ; X_{\xi}\right)$.

As the stability conditions, the solutions $\hat{U}_{\xi}$ are assumed to satisfy the following conditions:

$$
\begin{gathered}
\sup _{0<\xi \leq \xi_{0}} \sup _{0 \leq t \leq T}\left\|A_{\xi}\left(\hat{U}_{\xi}(t)\right)^{\gamma} \hat{U}_{\xi}(t)\right\|_{X_{\xi}}<\infty, \\
\sup _{0<\xi \leq \xi_{0}} \sup _{0 \leq s<t \leq T} \frac{\left\|\hat{U}_{\xi}(t)-\hat{U}_{\xi}(s)\right\|_{Y_{\xi}}<\infty .}{(t-s)^{\gamma-\alpha}}<.
\end{gathered}
$$

We can now state our result concerning the estimate of discretization error.

Theorem 4.1. Let all the structural assumptions be satisfied. Let $U_{0}$ be an initial value satisfying (4.14), and let $\hat{U}_{0, \xi}$ be a family of initial values as (4.16). Assume that there exists a solution $U$ to (4.1) lying in (4.15) on an interval $[0, T]$, and that there exists a solution $\hat{U}_{\xi}$ to $(4.2)$ for each $0<\xi \leq \xi_{0}$ on the same interval $[0, T]$ with the stability conditions (4.17) and (4.18). Then, it holds that 


$$
\begin{aligned}
\left\|\hat{U}_{\xi}(t)-U(t)\right\|_{Y} \leq C_{U, \hat{U}_{\xi}}\left\{\sup _{0 \leq t \leq T}\left\|\left(1-P_{\xi}\right) U(t)\right\|_{Y}\right. \\
\left.\quad+\left\|A_{\xi}\left(\hat{U}_{0, \xi}\right)^{\alpha}\left(\hat{U}_{0, \xi}-P_{\xi} U_{0}\right)\right\|_{X}+t^{\gamma-1} O_{\xi}^{1-\alpha}\right\}, \\
0<t \leq T, 0<\xi \leq \xi_{0},
\end{aligned}
$$

where $C_{U, \hat{U}_{\xi}}$ depends on the function norms of $U$ appearing in (4.15) and the stability constants in (4.17) and (4.18) but is independent of $\xi$.

Remark 4.1. In order to utilize this result for verifying (3.3) we have to know more precisely what contributes determining the constant $C_{U, \hat{U}_{\xi}}$. As will be observed in the proof of the theorem in the next section, $C_{U, \hat{U}_{\xi}}$ is determined by all the constants and exponents appearing in the structural assumptions, $T$, the following norms of $U$ :

$$
\begin{gathered}
\sup _{0<t \leq T} t^{1-\gamma}\|A(U(t)) U(t)\|_{X}, \\
\sup _{0<s<t \leq T} \frac{s^{1-\gamma+\sigma}\|A(U(t)) U(t)-A(U(s)) U(s)\|_{X}}{(t-s)^{\sigma}},
\end{gathered}
$$

and the two norms of $\hat{U}_{\xi}$ given by (4.17) and (4.18).

\section{Proof of Theorem 4.1}

By (4.9) and (4.18) it is seen that

$$
\left\|A_{\xi}\left(\hat{U}_{\xi}(t)\right)\left\{A_{\xi}\left(\hat{U}_{\xi}(t)\right)^{-1}-A_{\xi}\left(\hat{U}_{\xi}(s)\right)^{-1}\right\}\right\|_{\mathscr{L}\left(X_{\xi}\right)} \leq C_{\hat{U}_{\xi}}|t-s|^{\gamma-\alpha}, \quad 0 \leq s, t \leq T
$$

with some constant $C_{\hat{U}_{\xi}}>0$ independent of $\xi$. According to the theory of linear evolution equations (cf. $[18,19,20])$, there exists an evolution operator $\left\{U_{\xi}(t, s)\right\}_{0 \leq s \leq t \leq T}$ on $X_{\xi}$ for the family of operators $A_{\xi}\left(\hat{U}_{\xi}(t)\right)$. Furthermore the following estimates are well known. For every $0 \leq \theta \leq 1$,

$$
\begin{gathered}
\left\|A_{\xi}\left(\hat{U}_{\xi}(t)\right)^{\theta} U_{\xi}(t, s)\right\|_{\mathscr{L}\left(X_{\xi}\right)} \leq C_{\hat{U}_{\xi}}(t-s)^{-\theta}, \quad 0 \leq s<t \leq T, \\
\left\|A_{\xi}\left(\hat{U}_{\xi}(t)\right)^{\theta} U_{\xi}(t, s) A_{\xi}\left(\hat{U}_{\xi}(s)\right)^{-\theta}\right\|_{\mathscr{L}\left(X_{\xi}\right)} \leq C_{\hat{U}_{\xi}}, \quad 0 \leq s<t \leq T
\end{gathered}
$$

with some constant $C_{\hat{U}_{\xi}}>0$ independent of $\xi$. Similarly, for $0 \leq \theta<1$,

$$
\left\|U_{\xi}(t, s) A_{\xi}\left(\hat{U}_{\xi}(s)\right)^{\theta}\right\|_{\mathscr{L}\left(X_{\xi}\right)} \leq C_{\hat{U}_{\xi}, \theta}(t-s)^{-\theta}, \quad 0 \leq s<t \leq T .
$$

We now introduce the Cauchy problem for an evolution equation obtained by projecting (4.1) on $X_{\xi}$. Indeed, applying $P_{\xi}$ to (4.1), we have 


$$
\left\{\begin{array}{l}
\frac{d P_{\xi} U}{d t}+P_{\xi} A(U) U=P_{\xi} F(U), \quad 0<t \leq T, \\
P_{\xi} U(0)=P_{\xi} U_{0} .
\end{array}\right.
$$

The above problem combined with (4.2) then yields that

$$
\left\{\begin{array}{l}
\frac{d}{d t}\left(\hat{U}_{\xi}-P_{\xi} U\right)+A_{\xi}\left(\hat{U}_{\xi}(t)\right)\left(\hat{U}_{\xi}-P_{\xi} U\right) \\
\quad=\left\{P_{\xi} A(U) U-A_{\xi}\left(\hat{U}_{\xi}\right) P_{\xi} U\right\}+P_{\xi}\left\{F\left(\hat{U}_{\xi}\right)-F(U)\right\}, \quad 0<t \leq T, \\
\hat{U}_{\xi}(0)-P_{\xi} U(0)=\hat{U}_{0, \xi}-P_{\xi} U_{0} .
\end{array}\right.
$$

Using the evolution operator $U_{\xi}(t, s)$ introduced above, we represent $\hat{U}_{\xi}-U$ as

$$
\begin{aligned}
\hat{U}_{\xi}(t)-U(t)= & \left(P_{\xi}-1\right) U(t)+U_{\xi}(t, 0)\left\{\hat{U}_{0, \xi}-P_{\xi} U_{0}\right\} \\
& +\int_{0}^{t} U_{\xi}(t, s)\left\{P_{\xi} A(U(s))-A_{\xi}\left(\hat{U}_{\xi}(s)\right) P_{\xi}\right\} U(s) d s \\
& +\int_{0}^{t} U_{\xi}(t, s) P_{\xi}\left\{F\left(\hat{U}_{\xi}(s)\right)-F(U(s))\right\} d s .
\end{aligned}
$$

We shall next estimate $Y$-norms of all the terms in the right hand side. By (4.10) and (5.2) (with $\theta=\alpha$ ), we observe that

$$
\begin{aligned}
\left\|U_{\xi}(t, 0)\left\{\hat{U}_{0, \xi}-P_{\xi} U_{0}\right\}\right\|_{Y} \leq & \hat{D}\left\|A_{\xi}\left(\hat{U}_{\xi}(t)\right)^{\alpha} U_{\xi}(t, 0) A_{\xi}\left(\hat{U}_{\xi}(0)\right)^{-\alpha}\right\|_{\mathscr{L}\left(X_{\xi}\right)} \\
& \times\left\|A_{\xi}\left(\hat{U}_{0, \xi}\right)^{\alpha}\left(\hat{U}_{0, \xi}-P_{\xi} U_{0}\right)\right\|_{X} \\
\leq & C_{\hat{U}_{\xi}}\left\|A_{\xi}\left(\hat{U}_{0, \xi}\right)^{\alpha}\left(\hat{U}_{0, \xi}-P_{\xi} U_{0}\right)\right\|_{X} .
\end{aligned}
$$

Similarly, by (4.5) and (5.1) (with $\theta=\alpha$ ),

$$
\begin{aligned}
& \left\|\int_{0}^{t} U_{\xi}(t, s) P_{\xi}\left\{F\left(\hat{U}_{\xi}(s)\right)-F(U(s))\right\} d s\right\|_{Y} \\
& \quad \leq \hat{D}\left\|P_{\xi}\right\|_{\mathscr{L}(X)} \int_{0}^{t}\left\|A_{\xi}\left(\hat{U}_{\xi}(t)\right)^{\alpha} U_{\xi}(t, s)\right\|_{\mathscr{L}\left(X_{\xi}\right)}\left\|F\left(\hat{U}_{\xi}(s)\right)-F(U(s))\right\|_{X} \\
& \quad \leq C_{\hat{U}_{\xi}} \int_{0}^{t}(t-s)^{-\alpha}\left\|\hat{U}_{\xi}(s)-U(s)\right\|_{Y} d s .
\end{aligned}
$$

The third term in the right hand side of (5.4) is estimated by the lemma below. In this way, we arrive at an integral inequality of the form

$$
\begin{aligned}
p(t) \leq C_{U, \hat{U}_{\xi}}[ & t^{1-\gamma}\left\|\left(1-P_{\xi}\right) U(t)\right\|_{Y}+t^{1-\gamma}\left\|A_{\xi}\left(\hat{U}_{0, \xi}\right)^{\alpha}\left(\hat{U}_{0, \xi}-P_{\xi} U_{0}\right)\right\|_{X} \\
& \left.+O_{\xi}^{1-\alpha}+\int_{0}^{t} t^{1-\gamma}(t-s)^{-\alpha} s^{2(\gamma-1)} p(s) d s\right]
\end{aligned}
$$


where

$$
p(t)=t^{1-\gamma}\left\|\hat{U}_{\xi}(t)-U(t)\right\|_{Y}, \quad 0 \leq t \leq T .
$$

First let us solve this in the case when $\gamma<1$. We rewrite as

$$
p(t) \leq C\left[t^{1-\gamma} A+B+\int_{0}^{t} \varphi(t, s) p(s) d s\right], \quad 0 \leq t \leq T,
$$

where

$$
\begin{aligned}
& A=\sup _{0 \leq t \leq T}\left\|\left(1-P_{\xi}\right) U(t)\right\|_{Y}+\left\|A_{\xi}\left(\hat{U}_{0, \xi}\right)^{\alpha}\left(\hat{U}_{0, \xi}-P_{\xi} U_{0}\right)\right\|_{X}, \\
& B=O_{\xi}^{1-\alpha}, \quad C=C_{U, \hat{U}_{\xi}}, \quad \varphi(t, s)=t^{1-\gamma}(t-s)^{-\alpha} s^{2(\gamma-1)} .
\end{aligned}
$$

For $0 \leq s \leq t$, we have

$$
\begin{aligned}
p(s) & \leq C\left[s^{1-\gamma} A+B+\int_{0}^{s} \varphi(s, \sigma) d \sigma \sup _{0 \leq \sigma \leq s} p(\sigma)\right] \\
& \leq C\left[t^{1-\gamma} A+B+C_{1} t^{\gamma-\alpha} \sup _{0 \leq s \leq t} p(s)\right] .
\end{aligned}
$$

Therefore,

$$
\left\{1-C C_{1} t^{\gamma-\alpha}\right\} \sup _{0 \leq s \leq t} p(s) \leq C\left\{t^{1-\gamma} A+B\right\} .
$$

This shows that, if $t$ is sufficiently small, say $0 \leq t \leq \varepsilon$, with some fixed $\varepsilon>0$ such that $C C_{1} \varepsilon^{\gamma-\alpha} \leq \frac{1}{2}$, then

$$
p(t) \leq \sup _{0 \leq s \leq t} p(s) \leq 2 C\left\{t^{1-\gamma} A+B\right\}, \quad 0 \leq t \leq \varepsilon .
$$

So let now $t>\varepsilon$. Then,

$$
\begin{aligned}
p(t) & \leq C\left[t^{1-\gamma} A+B+\int_{0}^{\varepsilon} \varphi(t, s) p(s) d s+\int_{\varepsilon}^{t} \varphi(t, s) p(s) d s\right] \\
& \leq C\left[t^{1-\gamma} A+B+2 C\left(\varepsilon^{1-\gamma} A+B\right) \int_{0}^{\varepsilon} \varphi(t, s) d s+T^{1-\gamma} \varepsilon^{2(\gamma-1)} \int_{\varepsilon}^{t}(t-s)^{-\alpha} p(s) d s\right] .
\end{aligned}
$$

After some calculations we obtain that

$$
p(t) \leq C_{2}\left[A+B+\int_{\varepsilon}^{t}(t-s)^{-\alpha} p(s) d s\right], \quad \varepsilon \leq t \leq T
$$

with some constant $C_{2}$. This inequality obviously yields that

$$
\begin{aligned}
p(t) \leq & C_{2}\left[A+B+C_{2}(A+B) \int_{\varepsilon}^{t}(t-s)^{-\alpha} d s\right. \\
& \left.+\int_{\varepsilon}^{t} \int_{\varepsilon}^{s}(t-s)^{-\alpha}(s-\sigma)^{-\alpha} p(\sigma) d \sigma d s\right] \leq C_{3}\left[A+B+\int_{\varepsilon}^{t}(t-\sigma)^{(1-\alpha)-\alpha} p(\sigma) d \sigma\right] .
\end{aligned}
$$


Repeating this procedure finite times, we can then arrive at a usual Gronwall's inequality. As a result we conclude that

$$
p(t) \leq C_{U, \hat{U}_{\xi}}(A+B), \quad \varepsilon \leq t \leq T .
$$

This together with (5.6) then shows that (4.19) is valid.

When $\gamma=1$, the integral inequality (5.5) reduces to (5.7) (with $\varepsilon=0$ ). Therefore, (4.19) is verified by the same argument as for (5.8).

Lemma 5.1. The third term is estimated by

$$
\begin{aligned}
& \left\|\int_{0}^{t} U_{\xi}(t, s)\left\{P_{\xi} A(U(s))-A_{\xi}\left(\hat{U}_{\xi}(s)\right) P_{\xi}\right\} U(s) d s\right\|_{Y} \\
& \quad \leq C_{U, \hat{U}_{\xi}}\left[t^{\gamma-1} O_{\xi}^{1-\alpha}+\int_{0}^{t}(t-s)^{-\alpha} s^{\gamma-1}\left\|\hat{U}_{\xi}(s)-U(s)\right\|_{Y} d s\right], \quad 0<t \leq T .
\end{aligned}
$$

Proof. We can write

$$
\begin{aligned}
\int_{0}^{t} U_{\xi}(t, s)\left\{P_{\xi} A(U(s))-A_{\xi}\left(\hat{U}_{\xi}(s)\right) P_{\xi}\right\} U(s) d s \\
=\int_{0}^{t} U_{\xi}(t, s) A_{\xi}\left(\hat{U}_{\xi}(s)\right)\left\{A_{\xi}\left(\hat{U}_{\xi}(s)\right)^{-1}-A_{\xi}(U(s))^{-1}\right\} P_{\xi} A(U(s)) U(s) d s \\
\quad+\int_{0}^{t} U_{\xi}(t, s) A_{\xi}\left(\hat{U}_{\xi}(s)\right)\left\{A_{\xi}(U(s))^{-1} P_{\xi} A(U(s))-1\right\} U(s) d s \\
\quad+\int_{0}^{t} U_{\xi}(t, s) A_{\xi}\left(\hat{U}_{\xi}(s)\right)\left(1-P_{\xi}\right) U(s) d s=I+I I+I I I .
\end{aligned}
$$

Then, by (4.9), (4.15) and (5.1), we observe that

$$
\begin{aligned}
\left\|A_{\xi}\left(\hat{U}_{\xi}(t)\right)^{\alpha} I\right\|_{X} \\
\leq \int_{0}^{t}\left\|A_{\xi}\left(\hat{U}_{\xi}(t)\right)^{\alpha} U_{\xi}(t, s)\right\|_{\mathscr{L}\left(X_{\xi}\right)} \\
\quad \times\left\|A_{\xi}\left(\hat{U}_{\xi}(s)\right)\left\{A_{\xi}\left(\hat{U}_{\xi}(s)\right)^{-1}-A_{\xi}(U(s))^{-1}\right\}\right\|_{\mathscr{L}\left(X_{\xi}\right)}\|A(U(s)) U(s)\|_{X} d s \\
\leq C_{U, \hat{U}_{\xi}} \int_{0}^{t}(t-s)^{-\alpha} s^{\gamma-1}\left\|\hat{U}_{\xi}(s)-U(s)\right\|_{Y} d s .
\end{aligned}
$$

To estimate $I I$ and $I I I$ we utilize a formula

$$
U(s)=U(t)+A(U(s))^{-1} G(t, s), \quad 0 \leq s<t \leq T,
$$

where 


$$
\begin{aligned}
G(t, s)= & A(U(s))\left\{A(U(s))^{-1}-A(U(t))^{-1}\right\} A(U(t)) U(t) \\
& +\{A(U(s)) U(s)-A(U(t)) U(t)\} .
\end{aligned}
$$

By (4.4) and (4.15) (see also (1.3)), we verify that

$$
\|G(t, s)\|_{X} \leq C_{U}\left[(t-s)^{\gamma-\alpha} t^{\gamma-1}+(t-s)^{\sigma} s^{\gamma-\sigma-1}\right] .
$$

Then it follows that

$$
\begin{aligned}
I I= & \int_{0}^{t} U_{\xi}(t, s) A_{\xi}\left(\hat{U}_{\xi}(s)\right)\left\{R_{\xi}(U(s))-1\right\} A(U(s))^{-1} G(t, s) d s \\
& +\int_{0}^{t} U_{\xi}(t, s) A_{\xi}\left(\hat{U}_{\xi}(s)\right)\left\{R_{\xi}(U(s))-R_{\xi}(U(t))\right\} U(t) d s \\
& +\int_{0}^{t} U_{\xi}(t, s) A_{\xi}\left(\hat{U}_{\xi}(s)\right)\left\{R_{\xi}(U(t))-1\right\} U(t) d s=I I_{1}+I I_{2}+I I_{3} .
\end{aligned}
$$

By (4.7), (4.12), (5.1), (5.3) and (5.9), we have

$$
\begin{aligned}
\left\|A_{\xi}\left(\hat{U}_{\xi}(t)\right)^{\alpha} I I_{1}\right\|_{X} \leq & \int_{0}^{t}\left\|A_{\xi}\left(\hat{U}_{\xi}(t)\right)^{\alpha} U_{\xi}\left(t, \frac{t+s}{2}\right)\right\|_{\mathscr{L}\left(X_{\xi}\right)} \\
& \times\left\|U_{\xi}\left(\frac{t+s}{2}, s\right) A_{\xi}\left(\hat{U}_{\xi}(s)\right)^{1-\alpha}\right\|_{\mathscr{L}\left(X_{\xi}\right)}\left\|A_{\xi}\left(\hat{U}_{\xi}(s)\right)^{\alpha}\right\|_{\mathscr{L}\left(X_{\xi}\right)} \\
& \times\left\|\left\{R_{\xi}(U(s))-1\right\} A(U(s))^{-1}\right\|_{\mathscr{L}(X)}\|G(t, s)\|_{X} d s \\
\leq & C_{U, \hat{U}_{\xi}} O_{\xi}^{1-\alpha} \int_{0}^{t}\left[t^{\gamma-1}(t-s)^{\gamma-\alpha-1}+(t-s)^{\sigma-1} s^{\gamma-\sigma-1}\right] d s \\
\leq & C_{U, \hat{U}_{\xi}} t^{\gamma-1} O_{\xi}^{1-\alpha} .
\end{aligned}
$$

Similarly, by (4.7), (4.13), (4.15), (5.1) and (5.3),

$$
\begin{aligned}
\| A_{\xi}\left(\hat{U}_{\xi}(t)^{\alpha} I I_{2} \|_{X} \leq\right. & \int_{0}^{t}\left\|A_{\xi}\left(\hat{U}_{\xi}(t)\right)^{\alpha} U_{\xi}\left(t, \frac{t+s}{2}\right)\right\|_{\mathscr{L}\left(X_{\xi}\right)} \\
& \times\left\|U_{\xi}\left(\frac{t+s}{2}, s\right) A_{\xi}\left(\hat{U}_{\xi}(s)\right)^{1-\alpha}\right\|_{\mathscr{L}\left(X_{\xi}\right)}\left\|A_{\xi}\left(\hat{U}_{\xi}(s)\right)^{\alpha}\right\|_{\mathscr{L}\left(X_{\xi}\right)} \\
& \times\left\|\left\{R_{\xi}(U(s))-R_{\xi}(U(t))\right\} A(U(t))^{-1}\right\|_{\mathscr{L}(X)}\|A(U(t)) U(t)\|_{X} d s \\
\leq & C_{U, \hat{U}_{\xi}} O_{\xi}^{1-\alpha} \int_{0}^{t} t^{\gamma-1}(t-s)^{\gamma-\alpha-1} d s \leq C_{U, \hat{U}_{\xi}} t^{\gamma-1} O_{\xi}^{1-\alpha} .
\end{aligned}
$$


The term $I_{3}$ is seen to be equal to

$$
I_{3}=\left\{1-U_{\xi}(t, 0)\right\}\left\{R_{\xi}(U(t))-1\right\} A(U(t))^{-1} A(U(t)) U(t) .
$$

Therefore, by (4.7), (4.12) and (4.15),

$$
\left\|A_{\xi}\left(\hat{U}_{\xi}(t)\right)^{\alpha} I I_{3}\right\|_{X} \leq\left\|A_{\xi}\left(\hat{U}_{\xi}(t)\right)^{\alpha}\right\|_{\mathscr{L}\left(X_{\xi}\right)}\left\|I I_{3}\right\|_{X} \leq C_{U} t^{\gamma-1} O_{\xi}^{1-\alpha} .
$$

Estimate of $I I I$ is carried out in a quite similar way. We write

$$
\begin{aligned}
I I I= & \int_{0}^{t} U_{\xi}(t, s) A_{\xi}\left(\hat{U}_{\xi}(s)\right)\left(1-P_{\xi}\right) A(U(s))^{-1} G(t, s) d s \\
& +\int_{0}^{t} U_{\xi}(t, s) A_{\xi}\left(\hat{U}_{\xi}(s)\right)\left(1-P_{\xi}\right) U(t) d s=I I I_{1}+I I I_{2} .
\end{aligned}
$$

By (4.7), (4.11), (5.1), (5.3) and (5.9),

$$
\left\|A_{\xi}\left(\hat{U}_{\xi}(t)\right)^{\alpha} I I I_{1}\right\|_{X} \leq C_{U} t^{\gamma-1} O_{\xi}^{1-\alpha} .
$$

Meanwhile, $\mathrm{III}_{2}$ is written as

$$
I I_{2}=\left\{1-U_{\xi}(t, 0)\right\}\left(1-P_{\xi}\right) A(U(t))^{-1} A(U(t)) U(t),
$$

and is therefore estimated by

$$
\left\|A_{\xi}\left(\hat{U}_{\xi}(t)\right)^{\alpha} I I I_{2}\right\|_{X} \leq C_{U} t^{\gamma-1} O_{\xi}^{1-\alpha} .
$$

Thus we have accomplished the proof of the lemma.

\section{Application to chemotaxis-growth system}

We shall now apply our abstract results to the system (1.1). We assume the following conditions. The region $\Omega$ is a bounded domain in $\boldsymbol{R}^{2}$ at least $\Omega$ is a Lipschitz domain. The sensitivity function $\chi(\rho)$ is a real smooth function of $\rho \in(-\infty, \infty)$ with uniformly bounded derivatives up to the third order

$$
\sup _{-\infty<\rho<\infty}\left|\frac{d^{i} \chi}{d \rho^{i}}(\rho)\right|<\infty \quad i=1,2,3 .
$$

The growth function $f(u)$ is a real smooth function of $u \in(-\infty, \infty)$ such that $f(0)=0$ and

$$
f(u)=(-\mu u+v) u \quad \text { for sufficiently large }|u|
$$

with $\mu>0$ and $-\infty<v<\infty$.

Global Solutions. Global solutions are constructed by the usual techniques (cf. $[14,20,21])$. In the paper [13, Theorem 4.5], it was shown that, under (6.1) and (6.2), for any initial value 


$$
U_{0} \in \mathscr{K}_{\varepsilon}=\left\{\left(\begin{array}{c}
u_{0} \\
\rho_{0}
\end{array}\right) ; 0 \leq u_{0} \in H^{\varepsilon+1}(\Omega), 0 \leq \rho_{0} \in H^{\varepsilon+1}(\Omega)\right\},
$$

where $0<\varepsilon<\frac{1}{2}$ is an arbitrarily fixed exponent, (1.1) possesses a unique global solution in the function spaces:

$$
\left\{\begin{array}{l}
0 \leq u \in \mathscr{C}\left([0, \infty) ; H^{\varepsilon+1}(\Omega)\right) \cap \mathscr{C}^{1}\left((0, \infty) ; L^{2}(\Omega)\right) \cap \mathscr{C}\left((0, \infty) ; H^{2}(\Omega)\right), \\
0 \leq \rho \in \mathscr{C}\left([0, \infty) ; H^{\varepsilon+1}(\Omega)\right) \cap \mathscr{C}^{1}\left((0, \infty) ; L^{2}(\Omega)\right) \cap \mathscr{C}\left((0, \infty) ; H^{2}(\Omega)\right) .
\end{array}\right.
$$

From this it is also verified that if $S(t)$ is defined by $S(t) U_{0}=\left(\begin{array}{l}u(t) \\ \rho(t)\end{array}\right)$, then

$$
S(t) \text { is a continuous semigroup on } \mathscr{K}_{\varepsilon} \text { with norm }\|\cdot\|_{H^{\varepsilon+1}} \text {. }
$$

We therefore have a dynamical system $\left(S(t), \mathscr{K}_{\varepsilon},\left\{H^{\varepsilon+1}(\Omega)\right\}^{2}\right)$.

Exponential Attractor. In this paragraph, $\Omega$ is assumed to be of class $\mathscr{C}^{3}$, see however Remark 6.1 below. First of all, the system $\left(S(t), \mathscr{K}_{\varepsilon},\left\{H^{\varepsilon+1}(\Omega)\right\}^{2}\right)$ can be reduced to some subsystem whose phase space is a compact set. Indeed, according to [13, Proposition 5.1] and its remark, there exists an absorbing and positively invariant set $\mathscr{X}$ of $S(t)$ contained in

$$
\mathscr{X} \subset\left\{\left(\begin{array}{c}
u \\
\rho
\end{array}\right) ; 0 \leq u \in H^{2}(\Omega), 0 \leq \rho \in H^{2}(\Omega),\|u\|_{H^{2}}+\|\rho\|_{H^{2}} \leq C\right\} .
$$

Here, an absorbing set means that, for any bounded set $B \subset \mathscr{K}_{\varepsilon}$, there exists a certain time $t_{B}$ such that $S(t) B$ is contained in $\mathscr{X}$ for every $t \geq t_{B}$. Therefore, $\left(S(t), \mathscr{K}_{\varepsilon},\left\{H^{\varepsilon+1}(\Omega)\right\}^{2}\right)$ is reduced to the system $\left(S(t), \mathscr{X},\left\{H^{\varepsilon+1}(\Omega)\right\}^{2}\right)$.

Next we notice that the universal space containing $\mathscr{X}$ can be replaced by some larger one. To verify the squeezing property $((2.5)$ or $(2.6))$ of $S(t)$ we use the space

$$
X_{1}=\left\{\left(\begin{array}{c}
u \\
\rho
\end{array}\right) ; u \in L^{2}(\Omega), \rho \in H^{1}(\Omega)\right\} .
$$

Then, according to [13, Theorem 5.2], $S(t)$ is a continuous semigroup on $\mathscr{X}$ with respect to the norm $\|\cdot\|_{X_{1}}$, and, additionally, $S(t)$ enjoys the squeezing property in $X_{1}$. Therefore, we conclude that there exists an exponential attractor $\mathscr{M}$ for the system $\left(S(t), \mathscr{X}, X_{1}\right)$.

We use the universal space $X_{1}$ only for verifying the squeezing property of $S(t)$. But once an exponential attractor is constructed, it can be an exponential attractor even in some other larger universal spaces. In fact, hereafter we shall use the space

$$
X=\left\{\left(\begin{array}{c}
u \\
\rho
\end{array}\right) ; u \in L^{2}(\Omega), \rho \in L^{2}(\Omega)\right\}
$$


Then, without difficulty, $\mathscr{M}$ is shown to be an exponential attractor also for the system $(S(t), \mathscr{X}, X)$. By the definition of fractal dimension, its fractal dimension is exactly the same as in $\left(S(t), \mathscr{X}, X_{1}\right)$.

Remark 6.1. In the paper [13], $\Omega$ is assumed to be of class $\mathscr{C}^{3}$. The reason why such regularity is needed is to rewrite the equations in (1.1) as a semilinear abstract evolution equation in the product Hilbert space $X_{1}$ in verifying the squeezing property. But if we use the compact smoothing property (2.7) of $S(t)$, we need not this regularity and can construct an exponential attractor even in a convex domain, see [2].

Finite Element Method. Let us proceed to the approximation problem. Hereafter, $\Omega$ is a convex polygonal domain in $\boldsymbol{R}^{2}$. We also assume that $f(u)$ satisfies a stronger decay condition than (6.2):

$$
f(u)=-\hat{\mu} u^{3}+\hat{v} u \quad \text { for sufficiently large }|u|
$$

with some $\hat{\mu}>0$ and $-\infty<\hat{v}<\infty$. In view of the fact that every solution $u$ to the problem (1.1) remains in the set $\mathscr{X}$ which is a bounded set of $\mathscr{C}(\bar{\Omega})$ (see $(6.5)),(6.7)$ is not actually restrictive.

As a universal space, we use $X$ given by (6.6). With some fixed exponent $0<\varepsilon_{1}<\frac{1}{2}$, we also set

$$
Y=\left\{\left(\begin{array}{l}
u \\
\rho
\end{array}\right) ; u \in H^{\varepsilon_{1}+1}(\Omega), \rho \in H^{\varepsilon_{1}+1}(\Omega)\right\} .
$$

For $U=\left(\begin{array}{l}u \\ \rho\end{array}\right) \in Y$, we consider the following sesquilinear form

$$
\begin{aligned}
a\left(U ; U_{1}, U_{2}\right)= & a \int_{\Omega} \nabla u_{1} \cdot \nabla \overline{u_{2}} d x-\int_{\Omega} u \chi^{\prime}(\operatorname{Re} \rho) \nabla \rho_{1} \cdot \nabla \overline{u_{2}} d x \\
& +b \int_{\Omega} \nabla \rho_{1} \cdot \nabla \overline{\rho_{2}} d x, \quad U_{1}=\left(\begin{array}{c}
u_{1} \\
\rho_{1}
\end{array}\right), U_{2}=\left(\begin{array}{c}
u_{2} \\
\rho_{2}
\end{array}\right)
\end{aligned}
$$

defined on the product space $\left\{H^{1}(\Omega)\right\}^{2}$. Let $A(U)$ be the linear operator defined by the formula

$$
a\left(U ; U_{1}, U_{2}\right)=\left(A(U) U_{1}, U_{2}\right)_{X}, \quad U_{1} \in \mathscr{D}(A(U)), U_{2} \in X .
$$

For each $U \in Y, A(U)$ is a densely defined closed linear operator in $X$ with domain

$$
\mathscr{D}(A(U))=\left\{\left(\begin{array}{l}
u \\
\rho
\end{array}\right) ; u \in H^{2}(\Omega), \rho \in H^{2}(\Omega), \frac{\partial u}{\partial n}=\frac{\partial \rho}{\partial n}=0 \text { on } \partial \Omega\right\},
$$

which is independent of $U$. 
We also set $F$ as

$$
F(U)=\left(\begin{array}{c}
f(\operatorname{Re} u) \\
-c \rho+d u
\end{array}\right), \quad U=\left(\begin{array}{l}
u \\
\rho
\end{array}\right) \in Y .
$$

Then (1.1) is formulated as an abstract evolution equation

$$
\left\{\begin{array}{l}
\frac{d U}{d t}+A(U) U=F(U), \quad 0<t<\infty, \\
U(0)=U_{0}=\left(\begin{array}{l}
u_{0} \\
\rho_{0}
\end{array}\right)
\end{array}\right.
$$

in $X$.

We next formulate a semi discrete approximate problem. Let $\left\{\tau_{\xi}\right\}_{0<\xi \leq \xi_{0}}$ be a family of triangulations to $\Omega$ with the size parameter $\xi=\max \left\{d_{\sigma} ; \sigma \in \tau_{\xi}\right\}>$ 0 , where $\sigma$ denotes a triangle appearing in $\tau_{\xi}$ and $d_{\sigma}$ its diameter. We assume that $\left\{\tau_{\xi}\right\}_{0<\xi \leq \xi_{0}}$ is regular and satisfies the inverse assumption, that is

$$
\delta \xi \leq \rho_{\sigma} \leq d_{\sigma} \leq r_{\sigma} \leq \delta^{-1} \xi, \quad \sigma \in \tau_{\xi}
$$

holds with some positive constant $\delta>0$ independent of $\xi$, here $\rho_{\sigma}$ (resp. $r_{\sigma}$ ) denotes the radius of the incircle (resp. the circumcircle) of $\sigma$.

Let $\mathscr{C}_{\xi}(\bar{\Omega})$ is the space of trial functions subordinate to $\tau_{\xi}$, namely the space of continuous functions which are affine on each element $\sigma \in \tau_{\xi}$. Let

$$
X_{\xi}=\left\{\left(\begin{array}{l}
u \\
\rho
\end{array}\right) ; u \in \mathscr{C}_{\xi}(\bar{\Omega}), \rho \in \mathscr{C}_{\xi}(\bar{\Omega})\right\}
$$

$X_{\xi}$ being equipped with the induced norm of $X$. According to [11, Proposition 2.1], $X_{\xi}$ is embedded in $Y$ with continuous embedding (of course depending on $\xi)$. In fact, this is verified by a direct calculation estimating $H^{1+\varepsilon_{1}}$-norm

$$
\|u\|_{H^{1+\varepsilon_{1}}}^{2}=\|u\|_{L^{2}}^{2}+\sum_{j=1}^{2} \iint_{\Omega \times \Omega} \frac{\left|D_{j} u(x)-D_{j} u(y)\right|^{2}}{|x-y|^{2+2 \varepsilon_{1}}} d x d y
$$

for the functions in $\mathscr{C}_{\xi}(\bar{\Omega})$. When $X_{\xi}$ is equipped with $Y$-norm, it is denoted by $Y_{\xi}$. By $P_{\xi}$ we denote the orthogonal projection from $X$ onto $X_{\xi}$.

For $U \in Y$, we consider the sesquilinear form

$$
\begin{aligned}
a\left(U ; \hat{U}_{1}, \hat{U}_{2}\right)= & a \int_{\Omega} \nabla \hat{u}_{1} \cdot \nabla \overline{\hat{u}_{2}} d x-\int_{\Omega} u \chi^{\prime}(\operatorname{Re} \rho) \nabla \hat{\rho}_{1} \cdot \nabla \overline{\hat{u}_{2}} d x \\
& +b \int_{\Omega} \nabla \hat{\rho}_{1} \cdot \nabla \overline{\hat{\rho}_{2}} d x, \quad \hat{U}_{1}=\left(\begin{array}{c}
\hat{u}_{1} \\
\hat{\rho}_{1}
\end{array}\right), \hat{U}_{2}=\left(\begin{array}{c}
\hat{u}_{2} \\
\hat{\rho}_{2}
\end{array}\right)
\end{aligned}
$$

on $X_{\xi}$. From the sesquilinear form, $A_{\xi}(U)$ is defined by 


$$
A\left(U ; \hat{U}_{1}, \hat{U}_{2}\right)=\left(A_{\xi}(U) \hat{U}_{1}, \hat{U}_{2}\right), \quad \hat{U}_{1}, \hat{U}_{2} \in X_{\xi}
$$

Then, $A_{\xi}(U)$ is a bounded linear operator on $X_{\xi}$.

In addition, $F_{\xi}$ is defined by

$$
F_{\xi}(\hat{U})=P_{\xi} F(\hat{U}), \quad \hat{U} \in X_{\xi} .
$$

We can then formulate the corresponding approximate problem

$$
\left\{\begin{array}{l}
\frac{d \hat{U}_{\xi}}{d t}+A_{\xi}\left(\hat{U}_{\xi}\right) \hat{U}_{\xi}=F_{\xi}\left(\hat{U}_{\xi}\right), \quad 0<t<\infty, \\
\hat{U}_{\xi}(0)=\hat{U}_{0, \xi}
\end{array}\right.
$$

in $X_{\xi}$. We have already studied this problem in the previous paper [1]. Under (6.1) and (6.7), (6.10) possesses, for each $\hat{U}_{0, \xi} \in X_{\xi}$, a unique global solution such that

$$
\hat{U}_{\xi} \in \mathscr{C}^{1}\left([0, \infty) ; X_{\xi}\right) .
$$

In this way, (6.10) defines a semigroup $S_{\xi}(t)$ on $X_{\xi}$. Then, $\left(S_{\xi}(t), X_{\xi}, X\right)$ is a dynamical system for each $\xi$.

Phase Spaces. We assume that there exists an absorbing, positively invariant set $\mathscr{X}$ satisfying (6.5) and the dynamical system $(S(t), \mathscr{X}, X)$ possesses an exponential attractor $\mathscr{M}$. To apply Theorem 3.1, we have to set phase spaces $\mathscr{X}_{\xi}$ in a suitable way that (3.1) is valid.

Fix an exponent $\varepsilon_{2}$ such that $0<\varepsilon_{1}<\varepsilon_{2}<\frac{1}{2}$, and let $\alpha=\frac{\varepsilon_{1}+1}{2}$ and $\gamma=\frac{\varepsilon_{2}+1}{2}$. It is then clear that

$$
\frac{1}{2}<\alpha<\gamma<\frac{3}{4} .
$$

We introduce a third Banch space

$$
W=\left\{\left(\begin{array}{c}
u \\
\rho
\end{array}\right) ; u \in H^{\varepsilon_{2}+1}(\Omega), \rho \in H^{\varepsilon_{2}+1}(\Omega)\right\} .
$$

We see $X_{\xi} \subset W$, and when $X_{\xi}$ is equipped with $W$-norm, it is denoted by $W_{\xi}$.

Let $C$ be a constant given by

$$
C=\sup _{U \in \mathscr{X}}\|U\|_{W}<\infty .
$$

Similarly, let $L$ be given by

$$
L=\sup _{U_{0} \in \mathscr{X}} \sup _{0 \leq s<t<\infty} \frac{\left\|S(t) U_{0}-S(s) U_{0}\right\|_{Y}}{(t-s)^{\gamma-\alpha}}<\infty .
$$


Using these constants, we define the phase spaces $\mathscr{X}_{\xi} \subset X_{\xi}$ for $S_{\xi}(t)$ as follows. Indeed, for each $\xi, \mathscr{X}_{\xi}$ consists of all elements of $\hat{U}_{0, \xi} \in X_{\xi}$ which satisfy the following three conditions:

$$
\begin{gathered}
\sup _{0 \leq t<\infty}\left\|S_{\xi}(t) \hat{U}_{0, \xi}\right\|_{W_{\xi}} \leq C+1 \\
\sup _{0 \leq s<t<\infty} \frac{\left\|S_{\xi}(t) \hat{U}_{0, \xi}-S_{\xi}(s) \hat{U}_{0, \xi}\right\|_{Y_{\xi}}}{(t-s)^{\gamma-\alpha}} \leq L+1 ;
\end{gathered}
$$

$$
\hat{u}_{\xi}(t) \geq 0 \text { and } \hat{\rho}_{\xi}(t) \geq 0 \text { for every } t, \quad \text { where } S_{\xi}(t) \hat{U}_{0, \xi}=\left(\begin{array}{c}
\hat{u}_{\xi}(t) \\
\hat{\rho}_{\xi}(t)
\end{array}\right)
$$

By the definition, we clearly verify that $\mathscr{X}_{\xi}$ is positively invariant for $S_{\xi}(t)$; in other words, $S_{\xi}(t)$ is a semigroup on $\mathscr{X}_{\xi}$.

It is also clear that

$$
\bigcup_{0<\xi \leq \xi_{0}} \mathscr{X}_{\xi} \subset\left\{U=\left(\begin{array}{l}
u \\
\rho
\end{array}\right) \in W ;\|U\|_{W} \leq C+1, u \geq 0, \rho \geq 0\right\}=\mathscr{K}_{\varepsilon_{2}, C+1} .
$$

Then, since $\mathscr{K}_{\varepsilon_{2}, C+1}$ is a bounded set of $\mathscr{K}_{\varepsilon_{2}}$, there exists a time $t_{\varepsilon_{2}, C+1}>0$ such that

$$
S(t) \mathscr{K}_{\varepsilon_{2}, C+1} \subset \mathscr{X} \quad \text { for all } t \geq t_{\varepsilon_{2}, C+1} .
$$

Meanwhile the set

$$
\bigcup_{0 \leq t \leq t_{\varepsilon_{2}, C+1}} S(t) \mathscr{K}_{\varepsilon_{2}, C+1}
$$

is a compact set of $Y$, since $\mathscr{K}_{\varepsilon_{2}, C+1}$ is a compact set of $Y$ and since $S(t)$ is a continuous semigroup on $\mathscr{K}_{\varepsilon_{1}}$ with respect to the $Y$-norm (see (6.4)).

We are hence led to set a new phase space

$$
\overline{\mathscr{X}}=\left\{\bigcup_{0 \leq t \leq t_{\varepsilon_{2}, C+1}} S(t) \mathscr{K}_{\varepsilon_{2}, C+1}\right\} \cup \mathscr{X} .
$$

Clearly, $\bar{X}$ possesses the following properties:

(1) $\bar{X}$ is a compact set of $Y$ and hence of $X$;

(2) $\bar{X}$ is positively invariant for $S(t)$, in other words $(S(t), \bar{X}, X)$ is a dynamical system;

(3) $\mathscr{M}$ is an exponential attractor for $(S(t), \bar{X}, X)$ also;

(4) $\mathscr{X}_{\xi} \subset \overline{\mathscr{X}}$ for every $0<\xi \leq \xi_{0}$.

Condition (3.3). Since $\bar{X}$ is a compact set of $Y$, there is a constant $R$ such that 


$$
\bar{X} \subset\left\{U \in Y ;\|U\|_{Y}<R\right\}=K .
$$

For each $U \in K$, a linear operator $A_{k}(U)$ is defined by

$$
A_{k}(U) \tilde{U}=A(U) \tilde{U}+k \tilde{U}, \quad \tilde{U} \in \mathscr{D}(A(U)),
$$

where $A(U)$ is the linear operator already defined by (6.8) and $k$ is a sufficiently large constant which will be specified below. $F_{k}(\cdot)$ is a nonlinear operator defined by

$$
F_{k}(U)=F(U)+k U, \quad U \in K .
$$

Then, since

$$
A(U) U-F(U)=A_{k}(U) U-F_{k}(U), \quad U \in \mathscr{D}(A(U)),
$$

(1.1) is written as an abstract problem of the form (6.9) with $A(U)$ and $F$ replaced by $A_{k}(U)$ and $F_{k}$.

In the same way as above we can define from $A_{k}(U)$ and $F_{k}$ approximate operators $A_{k, \xi}(U)$ and $F_{k, \xi}$. Since

$$
A_{\xi}\left(\hat{U}_{\xi}\right) \hat{U}_{\xi}-F_{\xi}\left(\hat{U}_{\xi}\right)=A_{k, \xi}\left(\hat{U}_{\xi}\right) \hat{U}_{\xi}-F_{k, \xi}\left(\hat{U}_{\xi}\right), \quad \hat{U}_{\xi} \in X_{\xi},
$$

(6.10) is equivalent to a problem of the same form with $A_{\xi}\left(\hat{U}_{\xi}\right)$ and $F_{\xi}$ replaced by $A_{k, \xi}\left(\hat{U}_{\xi}\right)$ and $F_{k, \xi}$.

All the structural assumptions made above can then be verified provided that $k$ is sufficiently large. In fact Nakaguchi and the second author $[11,12]$ have studied fully discrete approximation for the local solutions to (1.1). As a matter of fact, the present structural assumptions are the same as in [11]; therefore we can appeal to the results obtained there.

From [11, Lemma 4.1], (4.3) is observed. Similarly, (4.4) follows from [11, Proposition 3.8]. (4.5) is verified directly. As shown in [11, Lemma 4.1], for $0 \leq \theta<\frac{3}{4}$

$$
\mathscr{D}\left(A_{k}(U)^{\theta}\right)=\left\{\left(\begin{array}{c}
u \\
\rho
\end{array}\right) ; u \in H^{2 \theta}(\Omega), \rho \in H^{2 \theta}(\Omega)\right\}, \quad U \in K .
$$

In particular $(\theta=\alpha),(4.6)$ is fulfilled. (4.7) follows from [11, Proposition 3.9] with $O_{\xi}=\xi^{2}$. (4.8) is observed from [11, Proposition 3.5]. Similarly (4.9) is observed from [11, Proposition 3.6]. As shown in [11, Lemma 4.3], for $0 \leq \theta<\frac{3}{4}$,

$$
\hat{C}_{\theta}^{-1}\left\|\hat{U}_{\xi}\right\|_{H^{2 \theta}} \leq\left\|A_{k, \xi}(U)^{\theta} \hat{U}_{\xi}\right\|_{X_{\xi}} \leq \hat{C}_{\theta}\left\|\hat{U}_{\xi}\right\|_{H^{2 \theta}}, \quad \hat{U}_{\xi} \in X_{\xi}, U \in K .
$$

Hence $(\theta=\alpha),(4.10)$ is verified. (4.11) is verifed from [11, Proposition 2.3]. Finally, (4.12) and (4.13) are verified from [11, Proposition 3.10]. 
In view of $(6.15)(\theta=\gamma)$, every $U_{0} \in \bar{X}$ satisfies the compatibility condition (4.14), and $S(t) U_{0}$ belongs to the space (4.15) with the estimates

$$
\sup _{0<t \leq 1} t^{1-\gamma}\left\|A_{k}\left(S(t) U_{0}\right) S(t) U_{0}\right\|_{X}+\sup _{1 \leq t<\infty}\left\|A_{k}\left(S(t) U_{0}\right) S(t) U_{0}\right\|_{X} \leq C
$$

and

$$
\begin{aligned}
& \quad \sup _{0<s \leq 1,0<s<t<\infty} \frac{s^{1-\gamma+\sigma}\left\|A_{k}\left(S(t) U_{0}\right) S(t) U_{0}-A_{k}\left(S(s) U_{0}\right) S(s) U_{0}\right\|_{X}}{(t-s)^{\sigma}} \\
& \quad+\sup _{1 \leq s<t<\infty} \frac{\left\|A_{k}\left(S(t) U_{0}\right) S(t) U_{0}-A_{k}\left(S(s) U_{0}\right) S(s) U_{0}\right\|_{X}}{(t-s)^{\sigma}} \leq C,
\end{aligned}
$$

where $C$ is independent of $U_{0} \in \overline{\mathscr{X}}$. These facts show that the norms for $S(t) U_{0}$ given by (4.20) and (4.21) are uniformly bounded with respect to $T$.

For every $\hat{U}_{0, \xi} \in \mathscr{X}_{\xi}$, (6.12) together with $(6.16)(\theta=\gamma)$ implies that

$$
\sup _{0 \leq t<\infty}\left\|A_{k, \xi}\left(S_{\xi}(t) \hat{U}_{0, \xi}\right)^{\gamma} S_{\xi}(t) \hat{U}_{0, \xi}\right\|_{X_{\xi}} \leq \hat{C}
$$

with some constant $\hat{C}$ independent of $\xi$. This result shows that the norm (4.17) for $S_{\xi}(t) \hat{U}_{0, \xi}$ is also uniformly bounded. Similarly, (6.13) implies that the norm (4.18) for $S_{\xi}(t) \hat{U}_{0, \xi}$ is uniformly bounded with respect to $T$.

We can now apply Theorem 4.1 to the functions $S_{\xi}(t) \hat{U}_{0, \xi}$ and $S(t) \hat{U}_{0, \xi}$ (with $U_{0}=\hat{U}_{0, \xi}$ ). In view of the Remark of Theorem 4.1, we immediately conclude that

$$
\left\|S_{\xi}(t) \hat{U}_{0, \xi}-S(t) \hat{U}_{0, \xi}\right\|_{Y} \leq C_{T} t^{\left(\varepsilon_{2}-1\right) / 2} \xi^{1-\varepsilon_{1}}, \quad 0<t \leq T
$$

for every $\hat{U}_{0, \xi} \in \mathscr{X}_{\xi}$ with some constant $C_{T}$ depending only on $T$. In particular,

$$
Q_{\xi}(T)=\sup _{\hat{U}_{\xi} \in X_{\xi}}\left\|S_{\xi}(T) \hat{U}_{\xi}-S(T) \hat{U}_{\xi}\right\|_{Y} \leq C_{T} \xi^{1-\varepsilon_{1}}, \quad 0<\xi \leq \xi_{0} .
$$

Finally we arrive at the main result of the paper. For any $\hat{U}_{\xi} \in \mathscr{X}_{\xi}$ and arbitrary time $0<T<\infty$,

$$
d\left(S_{\xi}(t) \hat{U}_{\xi}, \mathscr{M}\right) \leq \begin{cases}M e^{-\beta t}+C_{T} \xi^{1-\varepsilon_{1}} & \text { for } 0 \leq t \leq T \\ M e^{-\beta T}+C_{T} \xi^{1-\varepsilon_{1}} & \text { for } T \leq t<\infty\end{cases}
$$

\section{References}

[ 1 ] Aida, M. and Yagi, A., Global attractor for approximate system of chemotaxis and growth, Dynam. Conti. Discrete Impluls. Systems Series A 10 (2003), 309-315.

[2] Aida, M., Efendiev, M. and Yagi, A., Exponential attractor for quasilinear parabolic evolution equations, Osaka J. Math., to appear. 
[3] Alt, W. and Lauffenburger, D. A., Transient behavior of a chemotaxis system modelling certain types of tissue inflammation, J. Math. Biol. 24 (1985), 691-722.

[4] Budrene, E. O. and Berg, H. C., Complex patterns formed by motile cells of Escherichia coli, Nature 349 (1991), 630-633.

[ 5 ] Efendiev, M., Miranville, A. and Zelik, S., Exponential attractors for a nonlinear reactiondiffusion systems in $\boldsymbol{R}^{3}$, C. R. Acad. Sci. Paris 330 Série I (2000), 713-718.

[6] Ford, R. M. and Lauffenburger, D. A., Analysis of chemotactic bacterial distributions in population migration assays using a mathematical model applicable to steep or shallow attractant gradients, Bull. Math. Biol. 53 (1991), 721-749.

[7] Lauffenburger, D. A. and Kennedy, C. R., Localized bacterial infection in a distributed model for tissue inflammation, J. Math. Biol. 16 (1983), 141-163.

[8] Mimura, M. and Tsujikawa, T., Aggregating pattern dynamics in a chemotaxis model including growth, Physica A 230 (1996), 499-543.

[9] Mimura, M., Tsujikawa, T., Kobayashi, R. and Ueyama, D., Dynamics of aggregating patterns in a chemotaxis-diffusion-growth model equation, Forum 8 (1993), 179-195.

[10] Myerscough, M. R. and Murray, J. D., Analysis of propagating pattern in a chemotaxis system, Bull. Math. Biol. 54 (1992), 77-94.

[11] Nakaguchi, E. and Yagi, A., Fully discrete approximation by Galerkin Runge-Kutta methods for quasilinear parabolic systems, Hokkaido J. Math. 33 (2002), 385-429.

[12] Nakaguchi, E. and Yagi, A., Full discrete approximations by Galerkin method for chemotaxis-growth model, Proc. WCNA2000, Nonlinear Analysis 47 (2001), 6097-6107.

[13] Osaki, K., Tsujikawa, T., Yagi, A. and Mimura, M., Exponential attractor for a chemotaxisgrowth system of equations, Nonlinear Analysis 51 (2002), 119-144.

[14] Ryu, S.-U. and Yagi, A., Optimal control of Keller-Segel equations, J. Math. Anal. Appl. 256 (2001), 45-66.

[15] Tsujikawa, T., Singular limit analysis of planar equilibrium solutions to a chemotaxis model equation with growth, Methods Applications Anal. 3 (1996), 401-431.

[16] Tyson, R., Stern, L. G. and LeVeque, R. J., Fractional step methods supplied to a chemotaxis model, J. Math. Biol. 41 (2000), 455-475.

[17] Woodward, D. E., Tyson, R., Myerscough, M. R., Murray, J. D., Budrene, E. O. and Berg, H. C., Spatio-temporal patterns generated by Salmonella typhimurium, Biophys. J. 68 (1995), 2181-2189.

[18] Yagi, A., Parabolic evolution equations in which the coefficients are the generators of infinitely differentially semigroups, Funkcial. Ekvac. 32 (1989), 107-124.

[19] Yagi, A., Parabolic evolution equations in which the coefficients are the generators of infinitely differentially semigroups, II, Funkcial. Ekvac. 33 (1990), 139-150.

[20] Yagi, A., Abstract quasilinear evolution equations of parabolic type in Banach spaces, Boll. Un. Mat. Ital. 5-B (1991), 341-368.

[21] Yagi, A., Quasilinear abstract parabolic evolution equations with applications, "Evolution Equations, Semigroups and Functional Analysis", eds. A. Lorenzi and B. Ruf, Birkhäuser, Verlag Basel (2002), 381-397.

[22] Eden, A., Foias, C., Nicolaenko, B. and Temam, R., Exponential Attractors for Dissipative Evolution Equations, John Wiley \& Sons, Chichester, New York, 1994.

[23] Haken, H., Synergetics, An Introduction 3rd ed., Springer-Verlag, New York, Berlin, Heidelberg, 1983.

[24] Nicolis, G. and Prigogine, I., Self-Organization in Nonequilibrium System-From Dissipative Structure to Order through Fluctuations, John Wiley \& Sons, Chichester, New York, 1977. 
[25] Temam, R., Infinite-Dimensional Dynamical systems in Mechanics and Physics 2nd ed., Springer-Verlag, New York, Belin, Heidelberg, 1997.

nuna adreso:

Masashi Aida

Department of Applied Physics

Osaka University

Suita, Osaka 565-0871

Japan

Atsushi Yagi

Dapartment of Applied Physics

Osaka University

Suita, Osaka 565-0871

Japan

E-mail: yagi@ap.eng.osaka-u.ac.jp

(Ricevita la 7-an de marto, 2003)

(Reviziita la 10-an de junio, 2003) 